\title{
Éducation : du cosmopolitisme au globalisme
}

Education: from cosmopolitism to globalism

Educación : del cosmopolitismo al globalismo

\section{Pavel Zgaga}

Traducteur : Agence Ubiqus et Marie-José Sanselme

\section{(2) OpenEdition}

1 Journals

Édition électronique

URL : https://journals.openedition.org/ries/733

DOI : $10.4000 /$ ries.733

ISSN : 2261-4265

Éditeur

France Education international

\section{Édition imprimée}

Date de publication : 1 décembre 2009

Pagination : $39-50$

ISBN : 978-2-8542-0577-0

ISSN : 1254-4590

\section{Référence électronique}

Pavel Zgaga, "Éducation : du cosmopolitisme au globalisme », Revue internationale d'éducation de Sèvres [En ligne], 52 I décembre 2009, mis en ligne le 01 décembre 2012, consulté le 26 avril 2022 URL : http://journals.openedition.org/ries/733 ; DOI : https://doi.org/10.4000/ries.733

Ce document a été généré automatiquement le 26 avril 2022

(c) Tous droits réservés 


\title{
Éducation : du cosmopolitisme au globalisme
}

\author{
Education: from cosmopolitism to globalism \\ Educación : del cosmopolitismo al globalismo
}

\section{Pavel Zgaga}

Traduction : Agence Ubiqus et Marie-José Sanselme

\section{NOTE DE L'ÉDITEUR}

Traduit de l'anglais

Cet article aborde, du point de vue de l'histoire des idées sur un « monde meilleur », un sujet largement débattu : quels sont les effets de la progression de l'internationalisation et de la globalisation de l'éducation? Quelles mutations conceptuelles se cachent derrière les processus qui transforment sans doute la diversité dans le domaine de l'éducation en "un monde unifié » (un monde) et "une école »? Sommes-nous gagnants ou perdants dans ces processus? Plusieurs dilemmes sont associés à ce questionnement, mais il n'y a pas de réponse unique.

\section{L'éducation pour un « monde meilleur » : du cosmopolitisme au nationalisme}

2 L'essence du questionnement pourrait-être la suivante : la globalisation : est-ce bon ou mauvais? Si nous l'appliquons à la sphère de l'éducation, nous sommes censés répondre, par exemple, à une question : «une école » dans « un monde » - est-ce bon ou mauvais? Il n'est pas possible d'y répondre simplement sur la base d'impressions personnelles à un moment donné ; tout d'abord, c'est une question de considérations conceptuelles, de réflexion sur l'histoire et la culture, etc. C'est l'approche que nous 
aimerions suivre ici. Pour commencer, nous aborderons le problème de la manière suivante : l'éducation peut-elle contribuer en quoi que ce soit à un monde meilleur?

3 La croyance en un progrès continu vers un monde meilleur appartient à une longue tradition, de même que la conviction que le bien est associé à la connaissance et à la quête de la connaissance, c'est-à-dire à l'apprentissage. À l'âge des Lumières, des jalons particulièrement solides ont été posés sur cette voie historique. Un certain nombre d'érudits et d'activistes politiques de cette époque pourraient être évoqués, mais nous n'en citerons qu'un: Condorcet, dans son Esquisse d'un tableau historique des progrès de l'esprit humain (1793-1794). «Nous ferons remarquer comment une instruction plus universelle dans chaque pays, en donnant à un plus grand nombre d'hommes les connaissances élémentaires qui peuvent leur inspirer, et le goût d'un genre d'étude, et la facilité d'y faire des progrès, doit ajouter à ces espérances; combien elles augmentent encore, si une aisance plus générale permet à plus d'individus de se livrer à ces occupations, puisqu'en effet à peine, dans les pays les plus éclairés, la cinquantième partie de ceux à qui la nature a donné des talents reçoivent l'instruction nécessaire pour les développer; et qu'ainsi le nombre des hommes destinés à reculer les bornes des sciences par leurs découvertes devrait alors s'accroître dans cette même proportion.» Et aussi : « Nous montrerons combien cette égalité d'instruction, et celle qui doit s'établir entre les diverses nations, accéléreraient la marche de ces sciences ». ${ }^{1}$

4 Ce fragment pourrait peut-être provoquer aujourd'hui des commentaires cyniques mais il mérite beaucoup plus que cela. Il ne fait aucun doute que les tentatives humaines, au cours des deux derniers siècles, de passer du "siècle des Lumières " à un "âge éclairé » (Kant en 1784) se sont heurtées à de nombreux problèmes et contradictions. À ce stade, nos observations rencontrent un certain nombre de paradoxes et nous rapprochent de la "Dialectique de la raison "2 comme nous l'ont appris les auteurs du siècle dernier. Nous essaierons d'approfondir certaines de ces questions, en commençant par le fameux Traité de pédagogie de Kant (Über Pädagogik, 1803).

5 Kant est à l'évidence né à une époque heureuse : aujourd'hui, dit-il, « on commence à juger exactement et à apercevoir clairement ce qui constitue proprement une bonne éducation. $»^{3}$ Cela est étroitement lié à ce qu'il appelle «la perfection de la nature humaine » et à la " perfection de l'espèce humaine ». Ainsi, une compréhension claire du potentiel de l'éducation «nous découvre la perspective du bonheur futur de l'espèce humaine ». Kant a cependant identifié une fracture entre l'éducation actuelle (ou présente) et l'éducation possible dans l'avenir. Les parents apprennent à leurs enfants à s'adapter à "l'état présent ", alors qu'ils devraient plutôt être élevés "d'après un état meilleur, possible dans l'avenir, c'est-à-dire d'après l'humanité et de son entière destination ».

Quelques lignes plus loin, Kant utilise le concept de cosmopolitisme. Non seulement les «parents et les princes » éduquent leurs enfants simplement pour qu'ils s'adaptent aux conditions présentes - ils ne visent pas du tout au «bien du monde»! - mais leur compréhension de l'éducation est purement instrumentale. Par conséquent, ce n'est pas une vue étriquée et partielle, mais une vue universelle et cosmopolite - «le jugement des connaisseurs les plus éclairés »- qui doit servir de tremplin vers un avenir meilleur. Kant va même plus loin : les enfants « doivent se réjouir du progrès du monde, même s'il ne se fait pas à leur avantage ni à celui de leur pays ». 
7 Ces réflexions datent de plus de deux cents ans. Elles remontent à la période qui a précédé immédiatement la naissance des États-nations en Europe. Depuis le début du $\mathrm{XIX}^{\mathrm{e}}$ siècle, le terme « cosmopolitisme » n'a pas été employé plus souvent ; au contraire, il n'a été utilisé qu'à de rares reprises, principalement dans les cercles de l'intelligentsia, pour décrire une position individuelle, par exemple, en opposition aux concepts agressifs des nationalismes de tous ordres. En principe, il a été totalement exclu des discours politiques et idéologiques dominants et les différentes formes de totalitarismes ont toujours manifesté une très forte hostilité à son égard.

8 Le terme cosmopolitisme est aujourd'hui passé de mode. Néanmoins, il semble que nous soyons, aujourd'hui plus que jamais, des «citoyens du monde». Est-ce le cas? On pourrait contester vivement la façon dont j'ai traduit le mot, à partir de vieilles racines grecques. Peut-être ne sommes-nous pas plus « citoyens du monde » que Kant. Ce qui nous distingue de ses contemporains, c'est que nous sommes beaucoup plus "globalisés» : et non en tant que citoyens, mais en tant que consommateurs globalisés. Les termes « cosmos» et "globe » ont quelque chose en commun, mais une relation conceptuelle entre cosmo-politisme et globalisme semble hors de propos.

\section{De l'éducation dans l'État-nation à l'internationalisation de l'éducation}

9 À première vue, l'apparition des systèmes scolaires obligatoires dans les nouveaux États-nations du XIX ${ }^{\mathrm{e}}$ siècle peut apparaitre comme la réalisation des idées du siècle des Lumières et même des idées humanistes plus anciennes. L'éducation cosmopolite individualiste selon Kant a ouvert de nouvelles perspectives, mais elle a conduit au concept d'éducation nationale, qui s'est matérialisé dans la réalité de l'Europe du début du XIX ${ }^{e}$ siècle, sous le contrôle de l'État-nation. Le changement de concept est survenu dans un processus de transition complexe au tournant du siècle. Sur ce point, nous nous appuyons sur le travail de John Dewey, qui a analysé cette question selon une perspective non européenne dans Démocratie et Éducation (1915).

10 Il se réfère aux dichotomies susmentionnées et à la tradition d'une idée du développement libre et complet d'une personnalité « privée » qui est impossible sans la reconnaissance de la responsabilité «sociale ", de la discipline et de la subordination politique. D’après lui, l'État-nation a été créé comme "un intermédiaire entre la réalisation de la personnalité privée d'un côté et de l'humanité de l'autre " (Dewey, 2004). Les circonstances étaient caractérisées par "l'Allemagne, dans la génération engagée dans la lutte contre Napoléon pour l'indépendance nationale ». Il indique que «moins de vingt ans après, les successeurs de Kant, Fichte et Hegel, développaient l'idée que la fonction principale de l'État est éducative », et conclut : " Dans cet esprit, l'Allemagne a été le premier pays à mettre sur pied un système d'éducation public, universel et obligatoire ». Le modèle s'est rapidement répandu en Europe et au-delà ; pourtant, il a connu plusieurs modes de mise en œuvre nationale spécifiques.

11 Ce qui est déterminant, c'est la forme historique rattachée à l'école à l'aube de l'âge industriel : c'est « une école » pour tout le pays - « un pays »; pour le bien de la Nation, et non pour le bien du cosmopolitisme individualiste. On ne saurait faire découler cette forme simplement des idées des Lumières et de leurs successeurs philosophiques; ces derniers ont certes eu un impact important sur l'évolution historique, mais les 
processus réels ont été beaucoup plus complexes. Ainsi, dans leur histoire de l'alphabétisation en France, François Furet et Jacques Ozouf (1977) ont mis en garde contre un danger méthodologique: si nous n'établissons pas une distinction entre «l'idéologie de l'école » et « l'histoire de l'école », la seconde pourrait être déduite par spéculation des "idées révolutionnaires » et de la «mythologie de l'école ». François Furet et Jacques Ozouf mettent en avant une caractéristique qui n'est pas du tout incompatible avec l'analyse de Dewey : sur son chemin vers l'âge industriel, l'école (de l'État) se révèle comme la figure principale d'un pouvoir social illimité sur le bonheur d'un individu. Sous cette forme spécifique, l'État-nation poursuit son travail de contribution à un « monde meilleur ».

On trouve dans l'ouvrage de Dewey un autre commentaire important: «la nouvelle idée de l'importance de l'éducation pour le bien-être et le progrès de l'homme fut accaparée par les intérêts nationaux", tandis que "la science, le commerce et l'art transcendent les frontières nationales » (Dewey, 2004). Au milieu de la Première Guerre mondiale, au moment où la confrontation entre les États-nations atteignait son apogée, il nous rappelle une autre dichotomie : le bien-être et le progrès constituent « l'intérêt national " capital, mais leur réalisation ne peut se faire qu'avec des outils qui transcendent les « frontières nationales ». La science et les arts sont, de par leur nature même, "cosmopolites ", et non "nationaux ». D'une manière similaire, un commerce qui resterait confiné dans l'État national conduirait à une économie autarcique; par nature il est "global », si nous pouvons employer ce terme ici.

Les systèmes éducatifs nationaux sont les enfants des États-nations. Ils sont apparus au cours des deux derniers siècles et se sont développés avec une très grande diversité. Cette diversité résulte également de la politique et de l'administration d'un pays ou d'une région spécifique, ainsi que des traditions culturelles, religieuses, linguistiques, etc. La polarisation entre les États-nations, leur regroupement en blocs, la coopération économique et le protectionnisme ont eux aussi influé sur leurs caractéristiques.

Traditionnellement, les caractéristiques particulières des systèmes éducatifs nationaux ont été conservées jalousement comme des aspects de l'identité nationale. Dès que les États-nations sortent de l'isolement et que le besoin de coopération prévaut, ces caractéristiques particulières peuvent devenir des obstacles. L'éducation, par sa nature même, dépasse elle aussi les frontières nationales. Les potentiels en matière d'enseignement, de recherche et de créativité artistique ont toujours été dangereusement réduits lorsqu'un pays a décidé de fermer ses frontières. Cela n'est pas nouveau. Je rappelle ces vérités notoires pour souligner qu'elles ne renvoient pas nécessairement à la globalisation dans l'éducation telle que nous l'entendons aujourd'hui. Le point que nous avons maintenant atteint fait plutôt référence à l'internationalisation dans l'éducation, qui nous est familière depuis assez longtemps.

L'internationalisation de l'éducation subsume des liens et une coopération entre des systèmes différents qui peuvent rester différents. Les motivations ont toujours été variées. Aux raisons universitaires ou culturelles de la coopération, il faut ajouter les raisons diplomatiques et politiques, ainsi que les raisons économiques. Au cours du siècle précédent, ces dernières semblent avoir été prépondérantes. Ces tendances se poursuivent aujourd'hui. Les relations plus « chaleureuses » entre les pays (c'est-à-dire les États-nations) ont été l'espace de coopération le plus largement couvert en matière d'éducation. 
16 En principe, l'internationalisation de l'éducation n'a pas été un processus pénible ou «menaçant»: elle a généralement ouvert de nouvelles perspectives et favorisé de nouveaux développements dans l'éducation nationale sans la mettre en danger. L'Étatnation reste entièrement responsable de la scolarisation dans le pays. Pourtant, vers la fin du siècle dernier, il a commencé à apparaître comme un obstacle à d'autres développements ou au moins comme un problème à résoudre. L'éducation a franchi les limites de l'éducation nationale.

\section{«Européanisation » de l'éducation}

17 Ce problème a été abordé de différentes manières au cours des deux ou trois dernières décennies, en raison de facteurs politiques, économiques et du monde des affaires, ainsi que de facteurs éducatifs et culturels. La "nouvelle histoire européenne", nous ne l'avons pas oublié, a commencé avec l'acier et le charbon, puis s'est poursuivie avec l'énergie atomique et l'économie dans son ensemble; enfin, des instances politiques communes ont été mises en place. Pendant longtemps, il n'y a pas eu de référence directe à l'éducation dans les traités légaux des Communautés; les sous-systèmes « soft » comme l'éducation et la culture restaient placés sous la responsabilité exclusive des États membres, c'est-à-dire des États-nations. Mais, souvenons-nous en, le Traité de Maastricht de 1992, incluait non seulement de nouvelles dispositions sur la défense, la justice et les affaires intérieures, mais également, et pour la première fois, sur l'éducation.

Il a été convenu que « l'action communautaire peut appuyer et compléter, si nécessaire, l'action des États membres tout en respectant pleinement la responsabilité des Étatsmembres pour le contenu de l'enseignement et l'organisation du système éducatif ainsi que leur diversité culturelle et linguistique » (article 126). La Communauté s'est donc vu accorder certaines responsabilités dans l'éducation, mais le principe de subsidiarité a été appliqué et les systèmes éducatifs nationaux restent en définitive placés sous la responsabilité des États membres. Une étape a été franchie par rapport à la forme traditionnelle de la coopération internationale en matière d'éducation.

Près de vingt ans se sont écoulés depuis et il est nécessaire de reconsidérer certains aspects du début des années 1990. Cette période fut marquée par une compétition économique mondiale croissante, largement liée au développement technologique. L'Europe de l'Ouest a alors été forcée de repenser sa position à l'échelle mondiale. D'un autre côté, ce fut une période de grands changements politiques en Europe de l'Est: dissolution d'une superpuissance, confusion généralisée, avec des guerres civiles dans plusieurs pays. C'est ici l'occasion de rappeler une « histoire de l'éducation » ancienne et mal connue.

20 Au début des années 1980 - une époque fort éloignée en apparence de celle où l'Europe et le monde entier ont pris conscience des menaces sérieuses et de l'éclatement inévitable de conflits dans les Balkans -, a eu lieu une réforme de l'éducation qui a partiellement contribué à l'effondrement de la Yougoslavie. Ce fut la dernière réforme de l'éducation menée par l'ancien régime et elle était confrontée à une double tâche : répondre aux défis du libéralisme politique et des mouvements de la société civile des années 1970, et, simultanément, adapter l'éducation aux «besoins de la société » de l'époque. Cette modernisation de l'éducation a été alors comprise dans le contexte d'un "socialisme à visage humain" et dans le cadre d'une terminologie politique - 
aujourd'hui totalement incompréhensible -, par lesquels la Yougoslavie socialiste s'est si nettement démarquée des autres pays du bloc de l'Est.

Dans la mesure où la dimension idéologique était un aspect important de la réforme, principalement en tant que réponse à la montée de l'opposition libérale intellectuelle, l'émergence d'une critique ouverte n'était plus qu'une affaire de temps. Elle est apparue pendant le processus de préparation d'un nouveau tronc commun de programmes au milieu des années 1980 : dans une société multiethnique, multilingue et multiculturelle, organisée politiquement en fédération et fortement décentralisée, le pouvoir "central», plus tard identifié comme le cercle de Slobodan Miloševi'c, n'exigeait rien de moins que "l'harmonisation » des matières scolaires telles que la langue maternelle, la littérature, l'histoire, etc. à travers toute la fédération. Il est important d'ajouter qu'à l'époque, l'éducation était supervisée au niveau des unités de la fédération (c'est-à-dire des "républiques socialistes »), et non au niveau fédéral. Par conséquent, une sorte de " principe de subsidiarité » était à l'œuvre.

Cette exigence provoqua immédiatement une violente révolte. Au moins dans mon pays, la Slovénie, elle est également devenue l'un des leviers de la renaissance de la société civile et du processus graduel de démocratisation qui ont abouti à l'indépendance en 1991. Les processus d'association et d'intégration combinés aux tendances globales de la scène européenne plus large ont ouvert beaucoup plus de perspectives et d'espoirs que les tentatives "d'harmonisation" forcée dans l'exYougoslavie. Phénomène étrange à cette époque, mais très réel : l'internationalisation (c'est-à-dire l'européanisation) semblait la meilleure garantie de la protection de l'identité culturelle des groupes ethniques et de leur diversité culturelle. Elle fut interprétée comme un élément de la lutte pour la défense des droits de l'homme et la démocratie. Dans un contexte de pressions auto-cratiques de plus en plus fortes et dans une atmosphère de tensions ethniques croissantes, la protection et le développement de l'éducation nationale (c'est-à-dire de l'éducation dans sa propre langue) n'était imaginable que dans un contexte international.

La morale de cette histoire? Tout d'abord, cela nous rappelle que l'éducation peut devenir l'otage des troubles politiques et des conflits militaires, alors que son importance pour le bien-être et le progrès de l'homme - $\mathrm{y}$ compris le bien-être et le progrès nationaux - exige une situation sociale et politique stable et démocratique, et autant que possible des "fenêtres ouvertes", pour laisser entrer "l'air frais " et l'inspiration. Par conséquent, et deuxièmement, l'éducation a désespérément besoin d'une constitution politique établie, d'une structure pour le maintien de l'équilibre, d'un cadre de droits fondamentaux qui rend l'éducation publique possible. Enfin, l'éducation publique doit avoir des fondements - juridiques et financiers - solides et stables, mais elle doit également conserver sa nécessaire autonomie : tant par rapport au gouvernement et/ou aux partis politiques que par rapport au marché et aux institutions financières.

24 L'Union européenne est devenue une entité qui dépasse largement une simple communauté de l'acier et du charbon. Il ne s'agit pas seulement d'une zone de libreéchange; les ambitions ont toujours été supérieures. Récemment, Anne Corbett a publié une « histoire » détaillée de la façon dont les universités ont figuré à l'agenda de l'Union européenne. Ce processus a reçu une impulsion décisive avec le projet Erasmus (1985-87) et a, au moins partiellement, suivi sa propre logique: la logique d'une « Europe éducative » face à « l'Europe technocratique ou économique » (Corbett, 2005). 
Une «Europe éducative " s'est développée au cours des deux décennies suivantes - une communauté d'étudiants, d'enseignants, de chercheurs, etc., qui coopèrent à travers les frontières et à travers les divisions du passé. C'est effectivement quelque chose de nouveau et une réalisation dont le vieux continent peut être fier. Dans les années 1990 déjà, le développement d'une coopération internationale en éducation, sous une forme encore jamais observée, a soulevé une nouvelle question qui rejaillit sur les autorités éducatives dans les différents pays. Questions ouvertes et problèmes liés à une mobilité croissante ont rendu nécessaire la recherche d'une convergence entre les divers systèmes éducatifs. Le cas le plus fameux est le Processus de Bologne. Même s'il est controversé et que ses principes se prêtent à différentes interprétations, il s'est agi d'un processus volontaire pour faire converger les systèmes, processus mené dans le cadre d'un partenariat, où les établissements scolaires, les associations universitaires et d'étudiants, ainsi que d'autres partenaires ont travaillé main dans la main avec les gouvernements.

Dans ce contexte, des questions «techniques » et d'autres plus substantielles ont été abordées. Avec le démarrage du Processus de Bologne, un point d'une portée considérable a été soulevé, à savoir que «l'Europe n'est pas seulement celle de l'euro, des banques et de l'économie ", mais qu' « elle doit être également une Europe de la connaissance " (Déclaration de la Sorbonne, 1998). Cette formulation - si l'on met de côté la question de savoir si elle était rhétorique ou plus profonde - a survécu pendant une décennie. Indépendamment de la diversité des commentaires, elle a contribué à maintenir une dimension importante pour la poursuite du débat.

Cette dimension est tout aussi importante pour l'Union européenne à 27 que pour la "grande " Europe; elle a également des conséquences majeures pour l'enseignement supérieur dans son ensemble. Dans une atmosphère caractérisée par le prochain élargissement de l'Union en 2004, la Commissaire européenne Viviane Reding a formulé cela d'une façon intéressante à l'occasion d'une conférence sur les droits en matière de culture et d'éducation dans l'Europe élargie : « Notre défi est de construire une Europe qui dépasse la sphère de l'économie pour favoriser le développement durable comme moyen de répondre aux attentes des citoyens concernant la qualité de vie et la diversité culturelle et sociale». Et plus loin: «Ce qui doit être réaffirmé, à l'aube de l'élargissement, c'est le rôle de la culture dans le développement d'une identité européenne sans laquelle l'Union serait condamnée à n'être rien de plus qu'une vaste zone de libre-échange » (in De Groof, 2005).

Où en sommes-nous aujourd'hui ? Sommes-nous parvenus «au-delà de la sphère de l'économie »? Ces objectifs rencontrent pour le moins un certain scepticisme, mais la question mériterait d'être réexaminée aujourd'hui.

\section{Éducation et globalisation}

28 L'enthousiasme qui a suivi la chute du mur de Berlin s'est calmé depuis un certain temps et un phénomène similaire s'est produit avec l'élargissement historique de l'Union européenne. Cette décennie a été marquée par la poursuite de la croissance et de la compétition économique globale. D'un autre côté, dans les "villages globaux » actuels, nous rencontrons des phénomènes qui étaient censés appartenir au passé : protectionnisme, exclusion, homophobie, intolérance, etc. 
Le terme " globalisation », inauguré il y a deux ou trois décennies et entré depuis dans le langage quotidien, a cessé d'être perçu comme une promesse pour devenir une menace. La globalisation en général, y compris la globalisation de l'éducation, a très probablement plus d'opposants aujourd'hui que de défenseurs dans l'opinion publique. Nous continuons cependant à acheter des articles bon marché fabriqués dans des pays lointains et à économiser de l'argent pour envoyer nos enfants dans une bonne université étrangère. Le mot "globalisation » est souvent considéré comme synonyme d'un certain nombre de termes similaires en apparence; c'est une source de nombreux malentendus. Il est donc important d'établir une distinction entre globalisation et internationalisation : la globalisation n'est pas l'internationalisation.

Ulrich Beck propose d'établir une distinction entre la globalisation en tant que concept analytique et le globalisme en tant qu'idéologie: "Pour moi, le globalisme est une vision du monde selon laquelle le marché mondial déplace ou remplace l'action politique; c'est l'idéologie $d u$ pouvoir $d u$ marché mondial, l'idéologie du néolibéralisme. C'est une vue monocausale et économiste qui réduit la multidimensionnalité de la globalisation à une dimension, la dimension économique (qui est également envisagée comme un processus linéaire) et qui ne formule d'autres dimensions - globalisation de l'écologie, de la culture, de la politique, de la société civile - que comme subordonnées au système du marché mondial, si elles sont formulées » (Beck, 1997).

31 En effet, l'histoire de l'humanité et de la civilisation pourrait être interprétée comme « l'histoire de la globalisation »: un processus d'accentuation de l'interdépendance, un processus de connectivité croissante à travers le monde. Les systèmes éducatifs nationaux traditionnels ainsi que les "espaces de l'enseignement» communs actuellement émergents (comme l'espace de l'enseignement supérieur européen, qui s'inspire du Processus de Bologne et doit être mis en place en 2010), les « espaces de la recherche » et les "espaces de la connaissance " peuvent être considérés comme des formes d'organisation répondant aux défis d'une phase donnée de la "globalisation ».

L'interdépendance a atteint à l'époque moderne un niveau inégalé. Tout au début d'une discussion sur la globalisation, Daniel Bell a remarqué que l'État-nation devenait « trop petit pour les gros problèmes et trop grand pour les petits problèmes de la vie » (Bell, 1987). Cette remarque pleine d'esprit pourrait également être appliquée à l'éducation : le rôle de l'État-nation dans l'éducation a changé. D'un côté, on insiste sur le fait que l'administration de l'éducation devrait être plus proche des communautés locales ou respecter entièrement l'autonomie institutionnelle. De l'autre, l'éducation figure non seulement dans le Traité de l'Union européenne, mais également dans la législation internationale. Souvenons-nous, par exemple, des conventions sur la reconnaissance des qualifications, qui sont si importantes non seulement pour les migrants et pour la mobilité des étudiants, mais qui contribuent au moins indirectement à établir la confiance mutuelle, l'amélioration de la qualité, la transparence des systèmes éducatifs et des dispositions légales, et, surtout, la convergence des systèmes.

Pour résumer : nous rencontrons des questions et des problèmes de nature globale et nous devons inventer des outils et des approches susceptibles de traiter efficacement les problèmes globaux. Les problèmes nationaux et locaux n'ont pas disparu et l'éducation continuera de servir les besoins des contextes sociaux, culturels et économiques spécifiques (c'est-à-dire les identités, les cultures, les professions, etc.), mais nous rencontrons également des problèmes qui dépassent l'importance locale ou 
nationale et qui ne peuvent être résolus qu'au niveau global (c'est-à-dire l'éducation pour la paix, les droits de l'homme, la tolérance, la protection de l'environnement, etc.).

Après avoir débattu de "la globalisation et l'éducation ", nous nous tournons à présent, comme le suggère Beck, vers l'éducation et le globalisme. Il y a dix ans, Thomas Friedman a soutenu que « la force motrice derrière la globalisation est le capitalisme du libre marché » (Friedman, 1999). Comme le titre de son livre l'indique, il faut également interpréter cette dernière comme la force motrice conduisant à un monde meilleur : la «Lexus » symbolise le chemin vers la prospérité et le développement (économiques), tandis que "l'olivier » symbolise le désir de conserver l'identité et les traditions. De tout temps, l'éducation a servi les deux pôles - prospérité et développement ainsi qu'identité et tradition -, et elle est aujourd'hui confrontée à un dilemme difficile : soit la prospérité soit la tradition. Le tournant du millénaire a été l'apogée du "plus grand boom économique de l'histoire ", comme on l'a souvent déclaré. La déréglementation, la privatisation et les marchés sont devenus des mots sacrés, apparemment sans alternative - pas d'alternative en tout cas lorsque le secteur public est remis en question. Tout cela a été présenté comme un chemin vers la prospérité, vers un « monde meilleur».

Il y a eu, bien sûr, des protestations, avec de vifs débats sur ces sujets. Les polémiques sur le GATS $^{4}$ et les luttes contre une "marchandisation » de l'éducation, dans la première moitié de cette décennie, illustrent parfaitement le champ de l'éducation. Malgré les protestations, les systèmes éducatifs ont été fortement affectés par le globalisme. Pratiquement partout, l'enseignement public a été remis en question et accusé d'être déficient en termes d'efficience et de qualité. Le terme de "valeurs » a été confisqué par l'économie et a pratiquement perdu toute connotation éthique. L'éducation " au bénéfice du client » (customer friendly) doit répondre aux « besoins du client » (customer needs) ; elle doit être basée sur le «droit de choisir » (right to choose) et, bien sûr, sur un " pouvoir d'achat " (purchasing power). Le discours d'une "nouvelle gestion publique » a étouffé les discours plus traditionnels - et l'école est devenue une « entreprise » (Laval, 2004).

On trouve dans la littérature contemporaine une liste d'autres propositions de ce type et une liste peut-être encore plus longue de critiques de ces dernières. De nombreux auteurs ont mis en garde contre une application exagérée et irréfléchie de la théorie de la «main invisible » à des domaines sociaux tels que l'éducation. La croyance que la «main invisible» coordonne au mieux les actions humaines et que la libre entreprise créera une vie "meilleure pour chacun", y compris pour ceux qui apparaissent aujourd'hui défavorisés, a été jugée sévèrement il y a des dizaines d'années. La principale critique aujourd'hui semble être la suivante: la théorie, lorsqu'elle est appliquée à l'ensemble de la vie sociale, rend, par exemple, la constitution politique et l'espace public ou les aides sociales et le bien public totalement redondants. L'école publique fait partie de cette redondance. Parallèlement au renforcement du pouvoir du marché mondial, nous assistons à une baisse de celle de l'État-nation.

L'an dernier, les signes indiquant la fin $\mathrm{du}$ "plus grand boom économique de l'histoire" se sont multipliés. La crise financière et ses effets sur l'économie, la politique et la société peuvent également toucher l'éducation. Rien ne sera jamais plus comme avant. Si l'on en croit des voix venues récemment d'outre-Atlantique, la crise a déjà eu des impacts majeurs sur l'éducation. «La plupart des commentaires sur les 
effets de la crise financière sur l'enseignement supérieur se sont focalisés sur le possible tarissement des prêts étudiants, " explique Arthur M. Hauptman, un consultant américain indépendant de Virginie. D'après lui, « l'effet de cette diminution des prêts sera sensible surtout pour ceux qui étudient dans les institutions privées aux tarifs élevés et les écoles de commerce à but lucratif. ${ }^{5}$ Est-ce l'occasion de réhabiliter l'idée de l'éducation publique - ou peut-être simplement un pas de franchi pour répercuter les remous de la crise du privé sur l'éducation publique?

Sur les rives européennes de l'Atlantique, ces dilemmes ne sont pas seulement hypothétiques. En février, des responsables d'universités se sont réunis à Prague, à l'occasion de la Convention annuelle de l'European University Association (EUA) pour débattre de deux sujets brûlants : l'avenir du Processus de Bologne et les effets de la crise financière sur l'enseignement supérieur. Chose intéressante, ils ont parlé du «brillant» avenir de l'enseignement supérieur européen en relation avec la crise financière. Aussi loin que nous puissions voir, il est difficile à l'heure actuelle de prévoir sérieusement ce qu'il adviendra de l'enseignement supérieur dans des prochains mois. L'EUA fait état de situations très variées dans l'ensemble de l'Europe: certains gouvernements annoncent et mettent en œuvre des réductions budgétaires dans le domaine de l'éducation, tandis que d'autres augmentent le niveau des fonds publics disponibles. Quel que soit le scénario, dans l'enseignement supérieur européen, qui se rapproche toujours plus de ses objectifs décennaux - un espace commun de l'enseignement supérieur en 2010 -, les objectifs des réformes les plus ambitieuses devront se confronter à des évolutions économiques très incertaines.

Finalement, nous revenons à la question : l'éducation peut-elle contribuer à un meilleur monde aujourd'hui ? À un certain degré, la question est rhétorique; nous n'avons entendu aucun argument mettant sérieusement en doute le lien entre le bien, la connaissance et l'éducation. Naturellement, il existe une polémique, et peut-être aussi une certaine confusion sur ce qu'il faut entendre par un "monde meilleur » et une "bonne éducation ». Et ce n'est pas la première fois dans l'histoire humaine.

Nous devrions peut-être poser la question différemment : comment l'éducation peutelle contribuer à un meilleur monde aujourd'hui ? Qu'est-ce qui empêche l'éducation d'y contribuer? Ma réponse est empruntée à Ulrich Beck : la "vue monocausale », qui réduit la multidimensionnalité de l'éducation à une seule dimension, la dimension économique. Pour clarifier : je suis le dernier à contester l'importance de l'éducation pour le développement économique. Ce point fait référence à une réduction sans fondement qui met en danger le concept d'éducation. Il est crucial de reconnaître un ensemble complet d'objectifs éducatifs, par exemple sous la forme que Jacques Delors a formulée clairement dans son fameux rapport à l'UNESCO (1996) : apprendre à savoir, apprendre à faire, apprendre à vivre ensemble et apprendre à être. 


\section{BIBLIOGRAPHIE}

BECK U. (1997): Was ist Globalisierung? Irrtümer des Globalismus - Antworten auf Globalisierung. Frankfurt am Main: Suhrkamp Verlag.

BELL D. (1987): “The World and the United States in 2013”, Daedalus 116/1987.

CONDORCET M. (1970) : Esquisse d'un tableau historique des progrès de l'esprit humain. (1793-1794). Texte revu et présenté par O.H. Prior. Nouvelle édition présentée par Yvon Belaval. Paris : Librairie philosophique J. Vrin, 1970, 247 pp. Édition électronique par Jean-Marie Tremblay : http://classiques.uqac.ca/ .

CORBETT A. (2005): Universities and the Europe of Knowledge. Ideas, Institutions and Policy Entrepreneurship in European Union Higher Education Policy, 1955-2005. Houndmills: Palgrave Macmillan.

DEWEY J. (2004): Democracy and Education. Mineola, New York: Dover Publications, Inc.

FURET F. and OZOUF J. (1977) : Lire et écrire : l'alphabétisation des Français de Calvin à Jules Ferry. vol. 1, 2. Paris: Minuit.

FRIEDMAN Th. L. (1999): The Lexus and the Olive Tree. New York: Farrar, Straus and Giroux.

KANT I. (1964): Über Pädagogik. Herausgegeben von D. F. Th. Rink. In: Werke, Bd. XII. Schriften zur Anthropologie, Geschichtsphilosophie, Politik und Pädagogik, 2; pp. 695-761. Frankfurt am Main: Suhrkamp Verlag.

LAVAL Ch. (2004) : L'école n'est pas une entreprise. Le néo-libéralisme à l'assaut de l'enseignement public. Paris : Editions La Découverte.

REDING V. (2002) : Forum 'Culture, Cultural Rights and Education in an Enlarged Europe'. In De Groof J. \& Lauwers G. (eds), Cultural and Educational Rights in the Enlarged Europe. Nijmegen : Wolf Legal Publishers, pp. 27-34.

\section{NOTES DE FIN}

1. Jean-Antoine-Nicolas Caritat, marquis de Condorcet, Esquisse d'un Tableau historique des progrès de l'esprit humain, « Dixième Époque », 1793-1794.

2. Dialektik der Aufklärung. Fragments philosophiques, par Max Horkheimer et Theodor W. Adorno (1944).

3. Toutes les traductions en français de Kant utilisées dans cet article sont de Jules Barni. Voir : http://filozofie.eu/fr/dmdocuments/kant_pedagogie.pdf (NdlR).

4. GATS (General Agreement on Trade in Services) : L'Accord général sur le commerce et les services est le premier ensemble de règles multilatérales, juridiquement contraignantes, qui régissent le commerce international des services. Cet accord est entré en vigueur le $1^{\mathrm{er}}$ janvier 1995 (NDLR).

5. Voir : http://www.bc.edu/bc_org/avp/soe/cihe/newsletter/Number54/p22_Hauptman.htm/. 


\section{RÉSUMÉS}

Il existe une croyance ancienne en un progrès continu vers un monde meilleur, qui se double de la conviction que le bien est associé au savoir et à la recherche du savoir, c'est-à-dire à l'apprentissage. Sur ces bases, il a été attendu de l'éducation qu'elle apporte une contribution significative à l'avènement d'un monde meilleur. L'article s'interroge sur cette contribution au regard des avancées de l'internationalisation et de la globalisation dans le monde contemporain. Le concept d'éducation est abordé à travers le cosmo-politisme du siècle des Lumières, le nationalisme des $\mathrm{XIX}^{\mathrm{e}}$ et $\mathrm{XX}^{\mathrm{e}}$ siècles, l'internationalisme du $\mathrm{XX}^{\mathrm{e}}$ siècle - en particulier le processus d'européanisation - et enfin à travers l'âge du globalisme que nous connaissons aujourd'hui. Contre une « vue monocausale » du globalisme, l'auteur appelle à la reconnaissance de toute une variété de finalités éducatives.

There has been a long tradition of belief in continuous progress towards a better world. There has also been a long tradition of a conviction that good is associated with knowledge and with striving for knowledge, i.e. with learning. On these bases, expectations have been made that education substantially contribute to a better world. The paper reconsiders this contribution from the aspect of the contemporary progressing internationalisation and globalisation of education. The concept of education is discussed in relation to the cosmopolitanism of the Enlightenment, the 19th and 20th century nationalism, the 20th century internationalism and in particular the process of "Europeanisation" and, finally, the contemporary age of globalism. Against the "monocausal view" of globalism the author reaffirms a recognition of a full range of educational purposes.

Existe una antigua creencia en un progreso continuo hacia un mundo mejor, reforzado por la convicción de que el bien se encuentra vinculado con el saber y la búsqueda del saber, es decir, el aprendizaje. Partiendo de dicha base, se había previsto que la educación aportase una importante contribución al nacimiento de un mundo mejor. El artículo analiza dicha contribución habida cuenta de los avances de la internacionalización y de la globalización en el mundo contemporáneo. El concepto de educación se estudia a través del cosmopolitismo del siglo de las Luces, el nacionalismo de los siglos XIX y XX, el internacionalismo del siglo XX (en concreto, el proceso de europeización) y por último, a través del globalismo que presenciamos actualmente. Frente a una "visión mono-causal" del globalismo, el autor exige el reconocimiento de una amplia variedad de finalidades educativas.

\section{INDEX}

Mots-clés : école, enseignement, finalité de l'éducation, inégalité sociale, mondialisation, pédagogie institutionnelle, sciences de l'éducation, valeur

Keywords : school, teaching, purpose of education, social inequality, institutional pedagogy, educational sciences, values, globalization

Palabras claves : escuela, enseñanza, finalidad de la educación, desigualdad social, pedagogía institucional, ciencias de la educación, valor, globalización

\section{AUTEURS}

\section{PAVEL ZGAGA}

Pavel Zgaga est Professeur des Universités et enseigne la philosophie de l'éducation à l'université de Ljubljana, Slovénie, où il a fondé le centre d'études des politiques éducatives qu'il préside. 
Ancien ministre de l'éducation de Slovénie, il a en outre piloté plusieurs projets de recherche internationaux en éducation. Il a également conduit des projets de développement de l'enseignement supérieur dans le contexte européen (en particulier dans le cadre du Processus de Bologne) ainsi qu'en matière de formation des enseignants du supérieur. 